\title{
Intraoperative C-arm CT for Assessment of Syndesmotic Reduction
}

\author{
Sandeep Patel ${ }^{1}$, Manjunath Nishani ${ }^{2}$, Sreedhara B Chaluvashetty ${ }^{3}$
}

\begin{abstract}
Anatomic syndesmotic reduction is an important predictor of successful outcomes after ankle fracture fixation with associated syndesmotic injury. Various methods to check syndesmotic reduction range from mini-open technique and visualization to various radiographic parameters on standard C-arm to postoperative CT scans. Intraoperative CT scan will definitely be the best imaging modality and can avoid re-surgeries. With the advent of C-arm CT scans, the whole process of intraoperative CT scans can be cost-effective, compact, and easy portability between operation theaters.

Keywords: Ankle fracture, Intraoperative CT, Syndesmosis injury.

Journal of Foot and Ankle Surgery (Asia Pacific) (2021): 10.5005/jp-journals-10040-1183
\end{abstract}

\section{INTRODUCTION}

Syndesmotic reduction is of paramount importance as any malreduction can grossly alter the congruity of the tibiotalar articulation, leading to early arthritis. Worse outcomes have been demonstrated when the syndesmosis is not anatomically reduced. ${ }^{1-5}$ Leeds and Ehrlich ${ }^{1}$ correlated syndesmosis malreduction with ankle arthritis at an average of 4 years after injury. Chissell and Jones ${ }^{2}$ noted functional results to be worse with increasing tibiofibular diastasis or malreduction; as per Weening and Bhandari, ${ }^{3}$ the only significant predictor of functional outcome was the accuracy of syndesmosis reduction. Weening and Bhandari ${ }^{3}$ and Gardner et al. ${ }^{4}$ reported tibiofibular malreduction rates of $16 \%$ and $24 \%$, respectively, with the use of standard radiographic criteria. Gardner et al. ${ }^{4}$ have shown in their study that many syndesmoses were malreduced on CT scan but went undetected by plain radiographs; they concluded that radiographic measurements alone do not accurately reflect the status of the distal tibiofibular joint and post-reduction radiographic measurements are inaccurate for assessing the quality of reduction. Sagi et al. ${ }^{5}$ have noted worse outcomes with syndesmotic malreductions at a 2-year follow-up and have advocated surgeons to perform open visualization of syndesmosis to achieve reduction. They advocated that a postoperative CT scan with comparison to the contralateral extremity may be essential to gauge reduction.

Intraoperative CT scan is a novel modality, not easily available at all centers. However, the availability of the same can help in guiding the best syndesmotic reduction intraoperatively and can avoid repeat surgeries. Immediate control of fracture and syndesmotic reduction and ideal screw positioning in high image quality can reduce the need for secondary revision surgery. There are limited case reports ${ }^{6,7}$ in literature which have used intraoperative CT scans and demonstrated their utility in achieving anatomic syndesmotic reduction. Intraoperative $\mathrm{CT}$ scans have assumed importance in spine surgeries and pelvic surgeries, ${ }^{8,9}$ where they can guide in adequate placement of screws. It can also be combined with navigation systems for improving accuracy.

During the past, intraoperative CT needed huge CT machines to be installed in the operation theaters which was very expensive and logistically difficult as they could not be shifted between operating rooms. However, at present, cone-beam computed
${ }^{1,2}$ Department of Orthopaedics, Postgraduate Institute of Medical Education and Research, Chandigarh, India

${ }^{3}$ Department of Radiodiagnosis and Imaging, Postgraduate Institute of Medical Education and Research, Chandigarh, India

Corresponding Author: Sandeep Patel, Department of Orthopaedics, Postgraduate Institute of Medical Education and Research, Chandigarh, India, Phone: +91 9901440404, e-mail: sandeepdrpatelortho@gmail. com

How to cite this article: Patel S, Nishani M, Chaluvashetty SB. Intraoperative C-arm CT for Assessment of Syndesmotic Reduction. J Foot Ankle Surg (Asia Pacific) 2021;8(4):209-210.

Source of support: Nil

Conflict of interest: None

tomography which can be acquired through flat-panel detectors has evolved as a breakthrough as this can be incorporated into $\mathrm{C}$-arms or $\mathrm{O}$-arms, which can make them mobile and can easily be shifted to different operation theaters. The advantage of flat-panel detector technology is high-image quality and lower dose when compared with conventional C-arms. The C-arm CT works on the principle of rotating and capturing multiple images after focusing on the area of interest. Thereafter, it creates a 3D volume-rendered image and multiple plane images (axial coronal and sagittal) can be assessed. Figures 1 and 2 depict a case of ankle fracture where intraoperative $\mathrm{CT}$ was used for assessing syndesmotic reduction.

Limitations of the above technology are its high cost, and its non-availability in all centers; hence, it is equally important to rely on other easily available methods to achieve perfect syndesmotic reduction. However, the $\mathrm{C}$-arm $\mathrm{CT}$ which works on 3D volume rendering is still less costly than conventional $C T$ scans and can be cost-effective with an added advantage of compactness and easy portability between operation theaters.

\section{CONCLUSION}

Intraoperative CT scans, an emerging imaging modality is probably the best evaluator of syndesmosis reduction and when available 

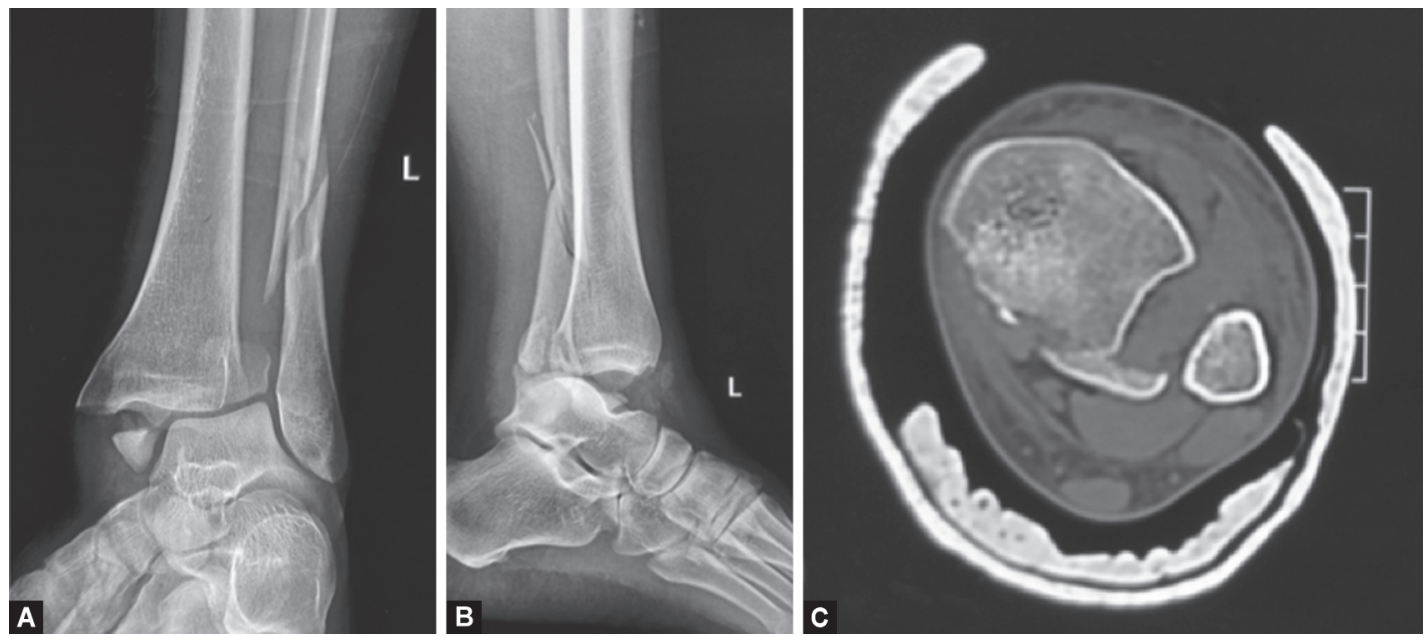

Figs $1 \mathrm{~A}$ to C: Preoperative X-rays (AP and lateral) of a trimalleolar fracture (PER 4 injury) and axial CT section depicting the syndesmotic injury
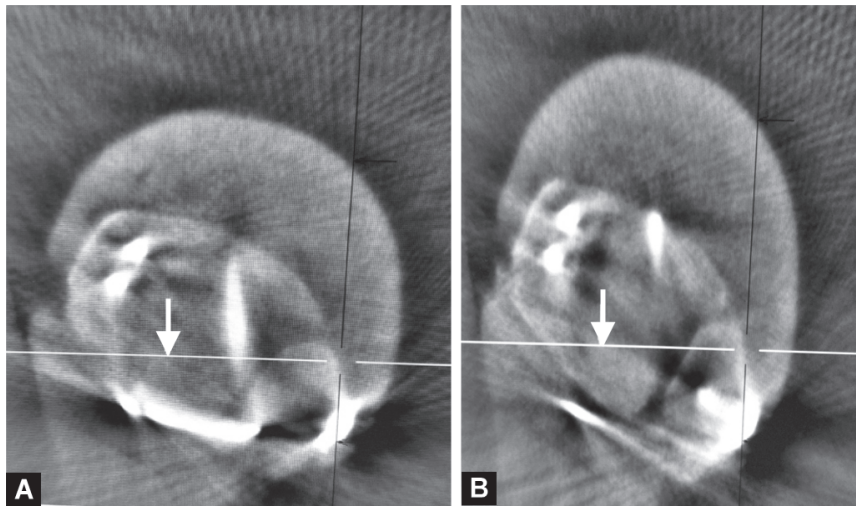

Figs $2 \mathrm{~A}$ and $\mathrm{B}$ : Intraoperative $\mathrm{CT}$ scan axial sections demonstrating anatomic syndesmosis reduction

should be used to confirm the syndesmosis reduction in complex ankle fractures.

\section{References}

1. Leeds HC, Ehrlich MG. Instability of the distal tibiofibular syndesmosis after bimalleolar and trimalleolar ankle fractures. J Bone Joint Surg Am 1984;66(4):490-503. DOI: 10.2106/00004623-198466040-00002.
2. Chissell HR, Jones J. The influence of a diastasis screw on the outcome of Weber type-C ankle fractures. J Bone Joint Surg Br 1995;77(3):435438. DOI: 10.1302/0301-620X.77B3.7744931.

3. Weening $B$, Bhandari M. Predictors of functional outcome following transsyndesmotic screw fixation of ankle fractures. J Orthop Trauma 2005;19(2):102-108. DOI: 10.1097/00005131-200502000-00006.

4. Gardner MJ, Demetrakopoulos D, Briggs SM, et al. Malreduction of the tibiofibular syndesmosis in ankle fractures. Foot Ankle Int 2006;27(10):788-792. DOI: 10.1177/107110070602701005.

5. Sagi HC, Shah AR, Sanders RW. The functional consequence of syndesmotic joint malreduction at a minimum 2-year follow-up. J Orthop Trauma 2012;26(7):439-443. DOI: 10.1097/ ВОT.0b013e31822a526a.

6. Hsu AR, Gross CE, Lee S. Intraoperative O-arm computed tomography evaluation of syndesmotic reduction: case report. Foot Ankle Int 2013;34(5):753-759. DOI: 10.1177/1071100712468872.

7. Summers HD, Sinclair MK, Stover MD. A reliable method for intraoperative evaluation of syndesmotic reduction. J Orthop Trauma 2013;27(4):196-200. DOI: 10.1097/BOT.0b013e3182694766.

8. Verma SK, Singh PK, Agrawal D, et al. O-arm with navigation versus C-arm: a review of screw placement over 3 years at a major trauma center. Br J Neurosurg 2016;30(6):658-661. DOI: 10.1080/02688697.2016.1206179.

9. Kinon $M D$, Desai $R$, Loriaux $\mathrm{D}$, et al. Image-guided percutaneous internal fixation of sacral fracture. J Clin Neurosci 2016;23:146-148. DOI: 10.1016/j.jocn.2015.08.011. 